ARTICLE

https://doi.org/10.1038/s41467-019-11902-6

\title{
Molecular ground-state dissociation in the condensed phase employing plasmonic field enhancement of chirped mid-infrared pulses
}

Ikki Morichika (1) ${ }^{1}$, Kei Murata (1) ${ }^{1}$, Atsunori Sakurai ${ }^{1}$, Kazuyuki Ishii ${ }^{1} \&$ Satoshi Ashihara (i) ${ }^{1}$

\begin{abstract}
Selective bond cleavage via vibrational excitation is the key to active control over molecular reactions. Despite its great potential, the practical implementation in condensed phases have been hampered to date by poor excitation efficiency due to fast vibrational relaxation. Here we demonstrate vibrationally mediated, condensed-phase molecular dissociation by employing intense plasmonic near-fields of temporally-shaped mid-infrared (mid-IR) pulses. Both down-chirping and substantial field enhancement contribute to efficient ladder climbing of the carbonyl stretch vibration of $\mathrm{W}(\mathrm{CO})_{6}$ in $n$-hexane solution and to the resulting $\mathrm{CO}$ dissociation. We observe an absorption band emerging with laser irradiation at the excitation beam area, which indicates that the dissociation is followed by adsorption onto metal surfaces. This successful demonstration proves that the combination of ultrafast optics and nano-plasmonics in the mid-IR range is useful for mode-selective vibrational ladder climbing, paving the way toward controlled ground-state chemistry.
\end{abstract}

\footnotetext{
${ }^{1}$ Institute of Industrial Science, The University of Tokyo, 4-6-1, Komaba, Meguro-ku, Tokyo 153-8505, Japan. Correspondence and requests for materials should be addressed to S.A. (email: ashihara@iis.u-tokyo.ac.jp)
} 
ctive control of chemical reactions on a molecular level, that is, selective breaking or making of chemical bonds, is one of the Holy Grails in physical chemistry ${ }^{1}$. Strong excitation of key reagent vibrations is a potentially powerful strategy for selective reaction $\operatorname{control}^{2}$, as has been validated by theoretical studies ${ }^{3-6}$. Vibrational motions may be activated through electronic excitation, but this scheme is restricted to modes accessible by Franck-Condon transitions and excited-state conical intersections ${ }^{7}$. In contrast, vibrational excitation via infrared (IR)/Raman processes at electronic ground states can be a more versatile technique due to its much broader accessibility to the potential energy surface. Compared with electronic excitation with ultraviolet/visible pulses, which causes various unwanted reactions, IR vibrational excitation is more advantageous for selectivity in reaction control.

Mid-IR ultrafast laser technologies have enabled mode-selective, multi-quantum vibrational excitation, and have opened a way to control molecular reactions at electronic ground states. Molecular dissociation mediated by vibrational ladder climbing (VLC) has been successfully achieved for gas-phase molecules ${ }^{8-11}$. Similar VLC has been performed on liquid-phase molecules as well ${ }^{12-16}$. Down-chirping proved useful for efficient VLC ${ }^{10,12,14}$, and arbitrary pulse-shaping was exploited for selective population of the excited vibrational levels ${ }^{15}$. These works have stimulated theoretical studies on the relevant topics ${ }^{17-19}$. However, attempts to observe ground-state dissociation in liquid phases ${ }^{12-16,20}$ have been unsuccessful to date, owing to the following reasons: collisions with solvent molecules accelerate vibrational relaxation to disturb deposition of sufficient energy on a specific mode, which would otherwise flow into the reaction coordinate, and dissociated species recombine on a short ( $\sim$ microsecond $)$ timescale.

We may resolve these issues to open an avenue toward controlled ground-state chemistry by combining ultrafast laser technology with plasmonics in the mid-IR range. Plasmonics takes advantage of coupling of light to metal electrons, enabling subwavelength localization and strong field enhancements. Therefore, surface-plasmon excitation substantially enhances light-matter interactions. Such enhancements in the mid-IR range have been utilized in surface-enhanced infrared absorption spectrosopy ${ }^{21-27}$. Recently, surface plasmons have been successfully applied to nonlinear vibrational spectroscopy ${ }^{28-32}$, where third-order nonlinear signals were amplified by the near-field enhancement of mid-IR pulses. Here, it is important to note that higher-order nonlinear processes are more significantly enhanced because of their higher-order dependence on field strengths ${ }^{33,34}$. This fact suggests that plasmonic field enhancement may play a key role in accelerating VLC to enable sufficient energy deposition on a timescale faster than vibrational relaxation. Recently, surface-enhanced VLC has been demonstrated for a paramercapto-benzonitrile adsorbed onto a rough metal surface but without any signs of reaction ${ }^{35}$.

In this paper, we realize the ground-state dissociation of condensed-phase molecules by employing chirped mid-IR pulses, substantially enhanced on a nanometer scale by plasmon excitation. In our reflection pump-probe experiments, mid-IR pump pulses are locally enhanced with plasmon excitation of gold nanoantennas to strongly drive VLC in the $T_{1 u}$ CO-stretching mode of $\mathrm{W}(\mathrm{CO})_{6}$ in $n$-hexane solution (Fig. 1). The resulting vibrational population distribution is sensitively probed as transient reflectance change, since population distribution is printed onto the reflectance spectrum of gold nanoantennas via antennamolecule coupling. Both down-chirping and near-field enhancements contribute to increasing the climbing efficiency and allow the observation of vibrational population up to $v=6$, whose energy is close to the CO-dissociation energy. Furthermore, we observe an absorption band emerging with mid-IR laser

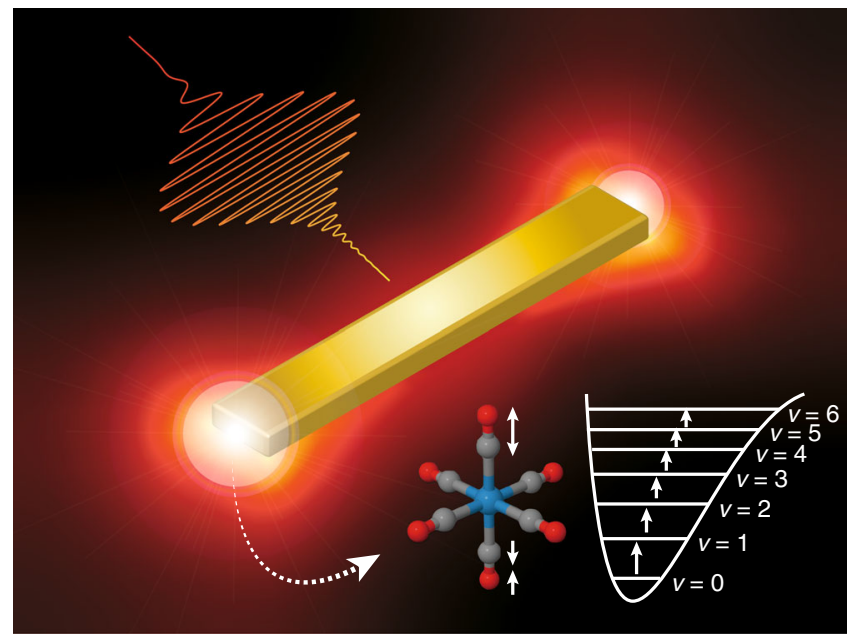

Fig. 1 Schematic view of the antenna-enhanced vibrational ladder climbing. The chirped mid-IR pulse, enhanced with plasmon excitation of the gold nanoantenna, strongly excites the carbonyl stretch vibration of $\mathrm{W}(\mathrm{CO})_{6}$ molecules

irradiation at the excitation beam area. These results show that the carbonyl compounds lose one CO ligand upon VLC, and the decarbonylated species are adsorbed onto the metal surfaces. The demonstrated scheme, where appropriately shaped mid-IR pulses are substantially enhanced in the vicinity of the metal surfaces, proved useful for mode-selective vibrational ladder climbing. This approach paves the way toward controlled ground-state chemistry in condensed phases, along with recent progress in other relevant topics ${ }^{36-40}$.

\section{Results}

$\mathrm{W}(\mathrm{CO})_{6} / \boldsymbol{n}$-hexane solution on gold nanoantenna arrays. Our sample is $\mathrm{W}(\mathrm{CO})_{6} / n$-hexane solution held between two $\mathrm{CaF}_{2}$ windows separated by a $25-\mu \mathrm{m}$ Teflon spacer (Fig. 2a). The target mode is the $T_{1 u}$ CO-stretching mode of $\mathrm{W}(\mathrm{CO})_{6}$, which exhibits an absorption at a center frequency of $1983 \mathrm{~cm}^{-1}$ with a fwhm linewidth of $3 \mathrm{~cm}^{-1}$. For one of the two $\mathrm{CaF}_{2}$ windows, the rodshaped gold nanoantennas are patterned into $2 \mathrm{D}$ arrays of rectangular lattice (see Methods for details). A scanning electron microscopy (SEM) image is shown in Fig. 2b. Figures $2 c$, d display the near-field distribution and the near-field enhancement spectrum of the nanoantenna arrays, respectively, simulated by the finite-difference time-domain (FDTD) method (see Supplementary Note 1 for details of the simulations). Here, we see that the nanoantennas exhibit localized surface-plasmon resonance at approximately the same frequency as the $T_{1 u}$ mode, with nearfield enhancements in the vicinity of the nanoantenna ends.

Antenna-molecule near-field coupling. The reflectance spectrum for the nanoantenna arrays immersed in $20 \mathrm{mMW}(\mathrm{CO})_{6} / n$ hexane solution is shown in Fig. 2e. The broad peak of the antenna resonance is observed to be strongly modified at the resonance frequency of the $T_{1 u}$ mode with spectral features characteristic to Fano resonance 24,25 . This modification is caused by coupling to the molecular vibration $\left(T_{1 u}\right.$ mode) in the following manner ${ }^{27}$. The incident field excites the plasmon mode, which in turn excites the molecular vibration via the near-field coupling. Next, the molecular vibrational excitation acts back to excite the plasmon mode. In this manner, coherent dipole excitation is continuously exchanged between the nanoantenna and the molecules until it is damped. The modification in the antenna 
a

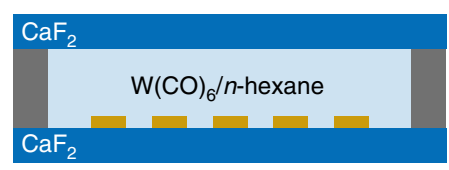

b
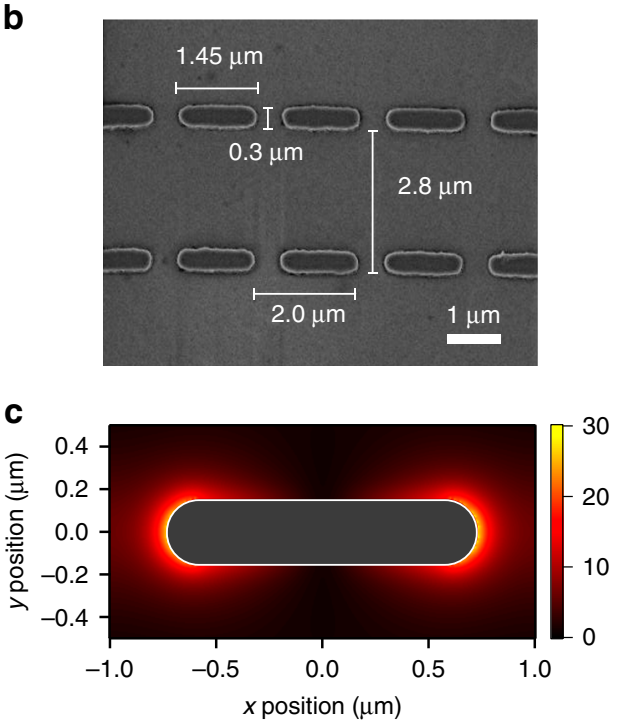

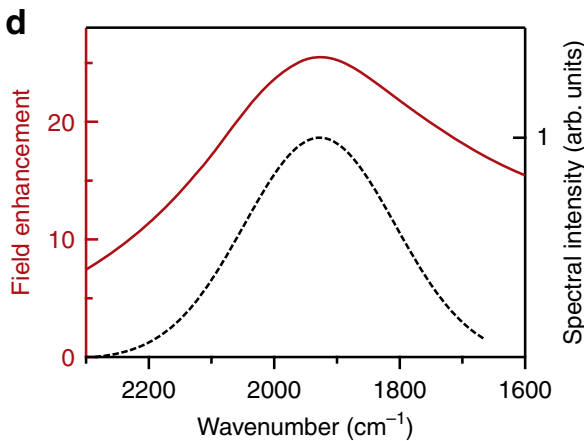

e

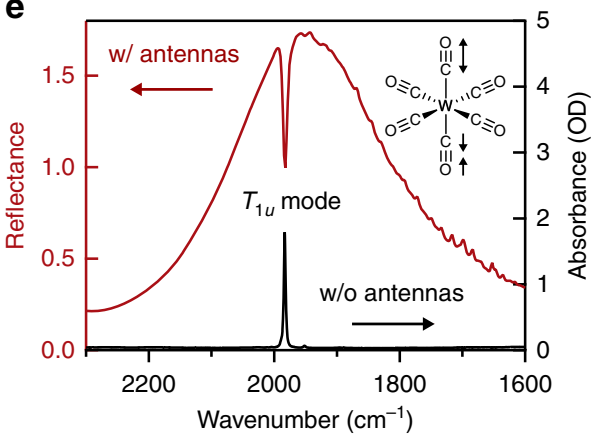

Fig. $2 \mathrm{~W}(\mathrm{CO})_{6} / n$-hexane solution on gold nanoantenna arrays. a A schematic of the sample comprising two $\mathrm{CaF}_{2}$ windows separated by a Teflon spacer, and filled with $\mathrm{W}(\mathrm{CO})_{6} / n$-hexane solution. $\mathbf{b}$ The SEM image of the gold nanoantenna arrays. Each nanoantenna is $1.45-\mu \mathrm{m}$ long, $0.3-\mu \mathrm{m}$ wide, and $0.1-\mu \mathrm{m}$ high. The size of the array unit cell is $2.0 \mu \mathrm{m} \times 2.8 \mu \mathrm{m}$. c Spatial distribution of the near-field enhancement factor simulated by the FDTD method. Dimensions of the nanoantenna are illustrated by a white line. $\mathbf{d}$ The simulated near-field enhancement factor in the vicinity of the nanoantenna ends (red) and the measured mid-IR laser pulse spectrum (black). e The measured reflectance for the nanoantenna arrays immersed in $20 \mathrm{mM} \mathrm{W}(\mathrm{CO})_{6} / n$-hexane solution (red) and the absorption spectrum for $3 \mathrm{mM} \mathrm{W}(\mathrm{CO})_{6} / n$-hexane solution (black)

reflectance results from such interference among direct/indirect plasmon excitations (see Supplementary Note 3 for details).

Transient spectroscopy of uncoupled $\mathbf{W}(\mathbf{C O})_{6}$. We perform mid-IR pump-probe spectroscopy for probing of VLC in the $T_{1 u}$ mode (see Methods and Supplementary Fig. 1 for a detailed description of the experimental setup). We use mid-IR pulses with a temporal duration of $100 \mathrm{fs}$, a center frequency of $1930 \mathrm{~cm}^{-1}$, and a repetition rate of $1 \mathrm{kHz}$. The fwhm bandwidth of $280 \mathrm{~cm}^{-1}$ provides spectral overlap with higher transitions of the anharmonic ladder. The linewidth of the antenna resonance is broad enough to cover the mid-IR pulse spectrum (Fig. 2d), which indicates that the enhanced near-field exactly follows the temporal waveform of the input pulse except for a $\pi / 2$-shift of the carrier phase (see Supplementary Note 1).

As a reference experiment, we measure the transient absorption of the uncoupled $\mathrm{W}(\mathrm{CO})_{6} / n$-hexane solution $(3 \mathrm{mM})$ in a transmission geometry using nearly Fourier-transform limited (FTL) pump pulses with a fluence of $0.2 \mathrm{~mJ} \mathrm{~cm} \mathrm{~cm}^{-2}$. Here, we report on the signal in terms of the absorbance change, $\Delta A=-\log _{10}\left(T / T_{0}\right)$, where $T$ and $T_{0}$ denote the probe transmittance with and without pumping, respectively. Figure 3 a shows the transient absorbance change at a 2 -ps time delay, which is long enough to avoid the pump-probe coherent artifacts. After excitation, the uncoupled molecules exhibit a negative absorption feature at the fundamental $T_{1 u}$ mode frequency (1982 $\left.\mathrm{cm}^{-1}\right)$, attributed to the ground-state bleach $(v=0 \rightarrow 1)$ and the stimulated emission $(v=1 \rightarrow 0)$. They also exhibit a positive absorption feature at $1967 \mathrm{~cm}^{-1}$, attributed to the excited-state absorption $(v=1 \rightarrow 2)$. These resonance frequencies are obtained by fitting the spectrum with a linear combination of Gaussian functions.

Transient spectroscopy of antenna-coupled $\mathrm{W}(\mathrm{CO})_{6}$. We measure the transient reflectance of the antenna-coupled $\mathrm{W}$ $(\mathrm{CO})_{6} / n$-hexane solution $(20 \mathrm{mM})$, and report on the signal in terms of the reflectance change, $\Delta R=R-R_{0}$, where $R$ and $R_{0}$ denote the probe reflectance with and without pumping, respectively. Figure $3 \mathrm{~b}$ shows the transient reflectance change at a 2-ps time delay, measured with the nearly-FTL pump pulses at a fluence of $0.2 \mathrm{~mJ} \mathrm{~cm}^{-2}$. After excitation, the antenna-coupled system exhibits a positive reflectance change at $1982 \mathrm{~cm}^{-1}$, attributed to the ground-state bleach $(v=0 \rightarrow 1)$ and the stimulated emission $(v=1 \rightarrow 0)$. They also exhibit negative reflectance changes, attributed to the excited-state absorptions: $v=1 \rightarrow 2$ at $1967 \mathrm{~cm}^{-1}, v=2 \rightarrow 3$ at $1953 \mathrm{~cm}^{-1}, v=3 \rightarrow 4$ at $1931 \mathrm{~cm}^{-1}$, $v=4 \rightarrow 5$ at $1908 \mathrm{~cm}^{-1}$, and $v=5 \rightarrow 6$ at $1889 \mathrm{~cm}^{-1}$. The sign inversion of the nonlinear signals, compared with the transmission measurements, is consistent with the fact that the peak feature observed for the uncoupled molecules in the linear absorbance manifests itself as the dip feature for the antennacoupled molecules in the linear reflectance (Fig. 2e). These resonance frequencies, obtained by fitting the reflectance change with a linear combination of Gaussian functions, agree well with the energy levels expected for a Morse potential given by

$$
E_{v}=\hbar \omega_{0}\left[\left(v+\frac{1}{2}\right)-\chi_{\mathrm{e}}\left(v+\frac{1}{2}\right)^{2}\right]
$$

Here, the anharmonicity of $\chi_{\mathrm{e}} \approx 0.0049$ and the corresponding anharmonic shift of $2 \chi_{\mathrm{e}} \omega_{0}=20 \mathrm{~cm}^{-1}$ (see Supplementary Fig. 3) 
a

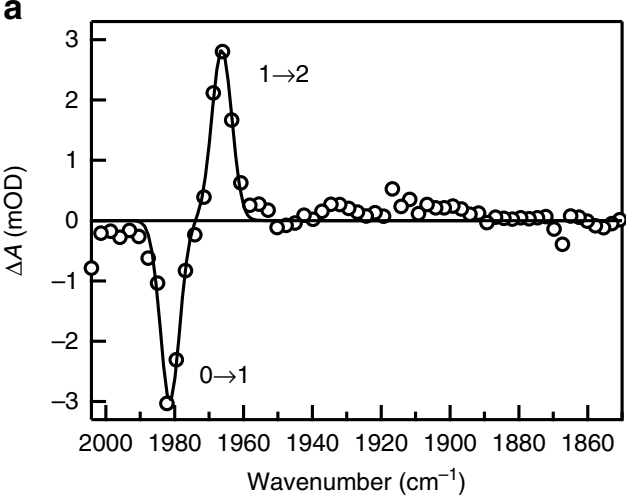

b

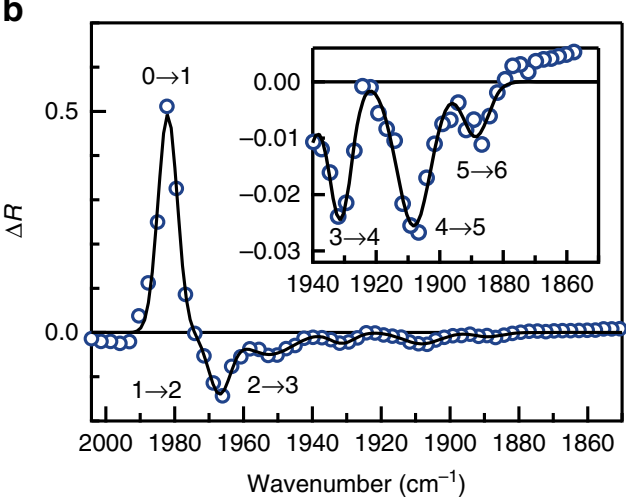

C

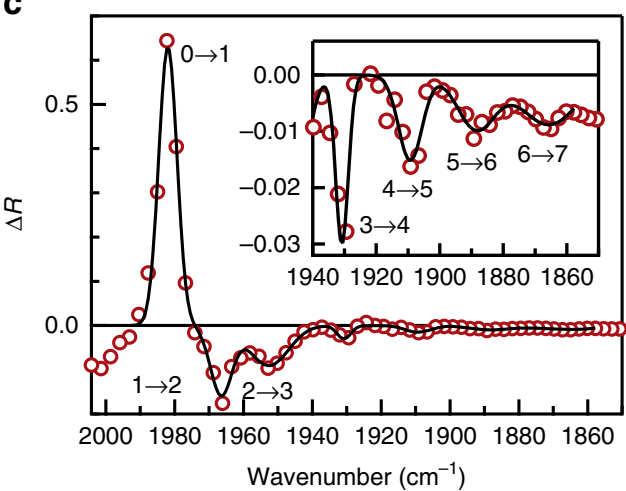

Fig. 3 Transient spectra for uncoupled/coupled $\mathrm{W}(\mathrm{CO})_{6}$ molecules. a The transient absorbance change of uncoupled $\mathrm{W}(\mathrm{CO})_{6}$ at a 2-ps time delay after excitation with nearly-FTL pulses. b, c The transient reflectance change of coupled $\mathrm{W}(\mathrm{CO})_{6}$ at a 2-ps time delay after excitation: $\mathbf{b}$ with nearly-FTL pulses, and $\mathbf{c}$ with down-chirped pulses. Each black line represents a linear combination of multiple Gaussian functions, fitted to the measured absorbance/reflectance change

are to be noted. These results are in line with those previously reported for uncoupled $\mathrm{W}(\mathrm{CO})_{6}$ molecules $^{13}$. Therefore, the signals observed in our transient reflection spectroscopy are certainly attributed to the vibrational excitation of $\mathrm{W}(\mathrm{CO})_{6}$ molecules. It is noteworthy that vibrational excitation up to $v=5$ is observed by employing plasmonic nanoantennas, despite the fact that the pump fluence is identical to that used for the uncoupled system shown in Fig. 3a.

Figure $3 c$ shows the transient reflectance change at a 2 -ps time delay measured with the down-chirped pump pulses (group-delay dispersion (GDD) of $-24000 \mathrm{fs}^{2}$ ) at a fluence of $0.2 \mathrm{~mJ} \mathrm{~cm} \mathrm{~cm}^{-2}$. Here, the temporal duration is stretched to $\sim 1.3 \mathrm{ps}$
(Supplementary Fig. 2e). In addition to the signals observed in Fig. 3b, we observe a signal at $1866 \mathrm{~cm}^{-1}$, attributed to the excited-state absorption of $v=6 \rightarrow 7$ transition. It indicates that the tungsten hexacarbonyl compounds are excited up to as high as $v=6$. In the previous study ${ }^{13}$ on liquid-phase $\mathrm{W}(\mathrm{CO})_{6}$, vibrational excitation up to $v=5$ was observed with nearly-FTL pump pulses at a fluence of $50 \mathrm{~mJ} \mathrm{~cm}^{-2}$. In this study, the irradiated pump fluence is as small as $0.2 \mathrm{~mJ} \mathrm{~cm} \mathrm{~cm}^{-2}$, but the pump fluence in the vicinity of nanoantennas is estimated to be $130 \mathrm{~mJ}$ $\mathrm{cm}^{-2}$, according to the simulated near-field enhancement of 25 as shown in Fig. 2c, d (comparison of VLC efficiency between experiments and density-matrix simulations suggests the effective near-field enhancement of $\sim 14$, as summarized in Supplementary Notes 2 and 3). Here, it is reasonably concluded that this large fluence as well as the down-chirping of the mid-IR pump pulses plays essential roles in realizing VLC up to the higher-lying states.

Emergence of a new band. We keep monitoring the pump-probe transient reflectance for the antenna-coupled molecules at a 2-ps time delay for an hour, while we irradiate the down-chirped pump pulses with GDD of $-24,000 \mathrm{fs}^{2}$ at a fluence of $0.2 \mathrm{~mJ} \mathrm{~cm} \mathrm{~cm}^{-2}$ with a repetition rate of $1 \mathrm{kHz}$. Figure $4 \mathrm{a}$ shows the transient reflectance change measured before and after an hour of pump irradiation. Note that the transient reflectance measured before the irradiation is essentially the same as the one shown in Fig. 3c. Surprisingly, the $1-h$ irradiation induces a change in the transient reflectance (also shown in Fig. 4a), i.e., there emerges an additional positive signal in the spectral range of $1910-1950 \mathrm{~cm}^{-1}$.

As shown in Fig. $4 \mathrm{~b}$, the transient reflectance change, $\Delta R$, at $1930 \mathrm{~cm}^{-1}$ monotonically increases with irradiation time. This indicates that the positive signal, which is superposed onto the signal intrinsic to the original $\mathrm{W}(\mathrm{CO})_{6}$ molecules, grow cumulatively with the irradiation of down-chirped pump pulses. In contrast, $\Delta R$ does not evolve with time when we irradiate the sample with nearly-FTL pump pulses with the same fluence of

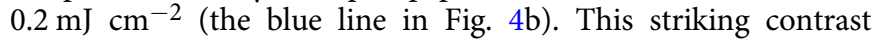
indicates the impact of down-chirping in emergence of the additional band.

Figure $4 c$ displays the transient reflectance change at a 2-ps time delay for varied irradiation time, subtracted by the one for 0 min, which clearly shows a cumulative evolution of the positive signal. By the Gaussian-fitting analysis on each spectrum, we find that the center frequency is blue-shifted, and the linewidth is narrowed with irradiation time, as shown in Fig. $4 \mathrm{~d}$. After $1 \mathrm{~h}$ of the pump irradiation, the positive signal exhibits a center frequency of $1930 \mathrm{~cm}^{-1}$ and a fwhm linewidth of $22 \mathrm{~cm}^{-1}$.

After the 1-h irradiation with the down-chirped pump pulses, we measure the linear reflectance for the sample area of $200 \mu \mathrm{m} \times$ $200 \mu \mathrm{m}$ inside/outside the irradiation beam spot (Pump: $200 \mu \mathrm{m}$, probe: $150 \mu \mathrm{m}$ ), shown in Fig. 4e. In addition to the original absorption dip of the $T_{1 u}$ mode at $1982 \mathrm{~cm}^{-1}$, an absorption dip is observed for the area inside the irradiation beam spot (not observed for the area outside the beam spot). Importantly, this dip exhibits the same center frequency and linewidth as the positive signal observed in the transient reflectance change with the pump irradiation. This coincidence indicates that the newlyappeared pump-probe signal shown in Fig. $4 a-d$ originates from the ground-state bleach and the stimulated emission of the vibrational mode with its fundamental resonance at $1930 \mathrm{~cm}^{-1}$. Note that the corresponding excited-state absorption is not clearly observed at frequencies below $1930 \mathrm{~cm}^{-1}$. The reason is not clear, but it is possible that population of this emerging vibrational mode is distributed over many excited levels because of efficient ladder climbing and that the resonant excited-state 

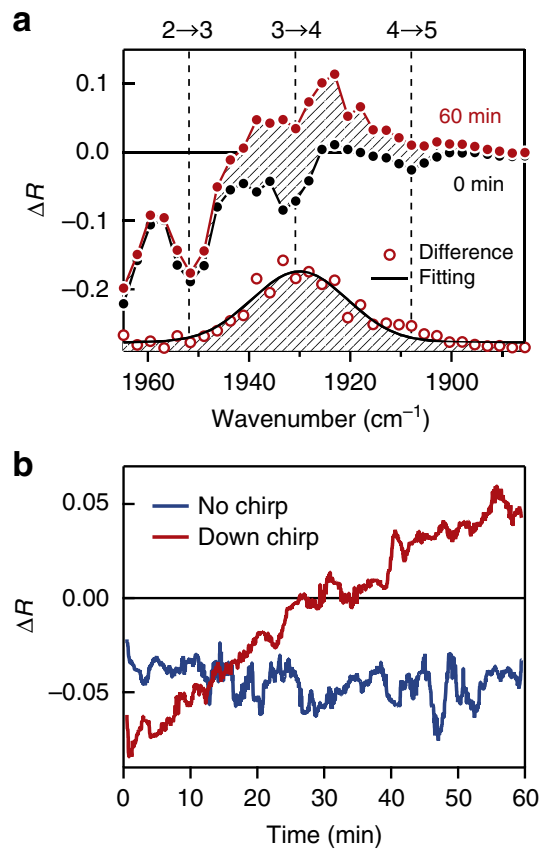

C

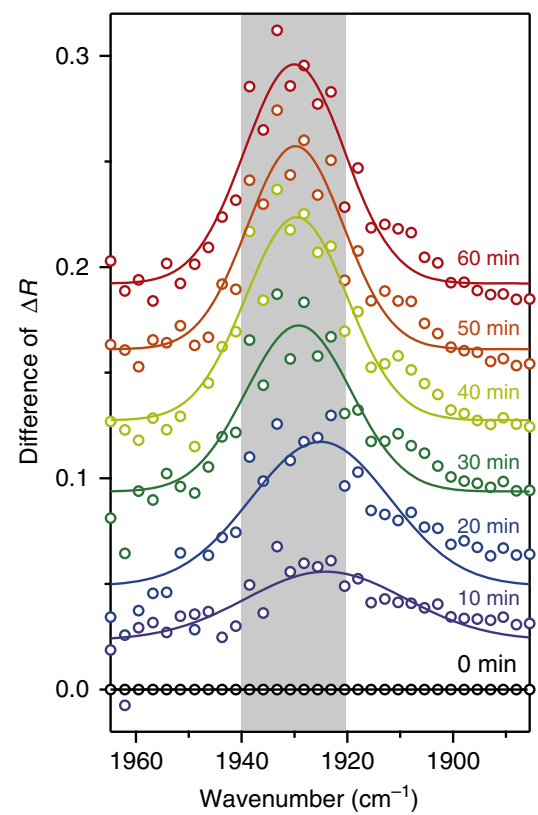

d
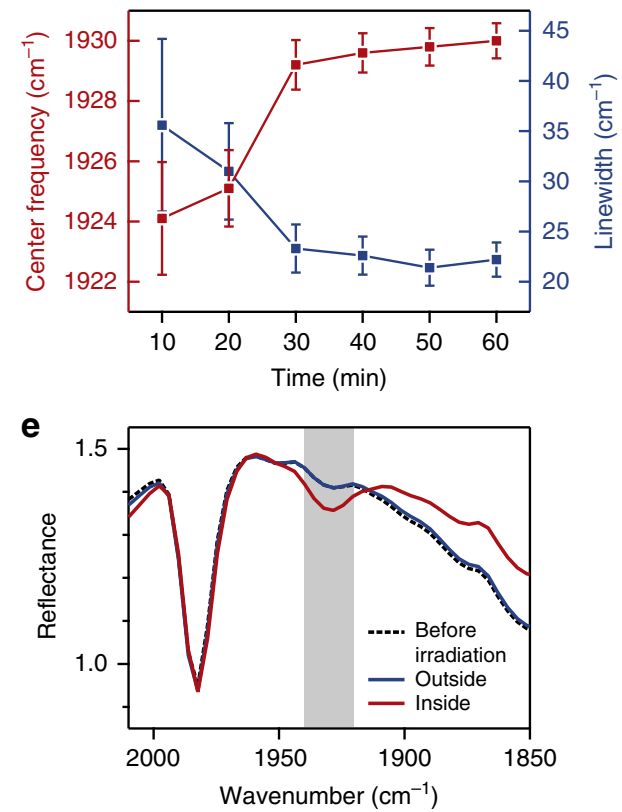

Fig. 4 Cumulative evolution of an absorption band with pump irradiation. a Transient reflectance change at a 2-ps time delay for the antenna-coupled W $(\mathrm{CO})_{6}$ before (black filled markers) and after (red filled markers) the 1-h irradiation with the down-chirped (GDD of $-24,000 \mathrm{fs}^{2}$ ) pump pulses at a fluence of $0.2 \mathrm{~mJ} \mathrm{~cm}-2$. The red open markers represent the difference between the two reflectance changes, and the black dashed line represents a Gaussian function fitted to the data. $\mathbf{b}$ Time evolution of the reflectance change at $1930 \mathrm{~cm}^{-1}$ during the repeated irradiation of pump pulses: nearly-FTL (blue) and down-chirped (red). c Transient reflectance change at a 2-ps time delay for varied irradiation time, subtracted by the one for 0 min. Solid lines represent Gaussian functions fitted to the data (markers). $\mathbf{d}$ Center frequency and linewidth of the emerging band at different irradiation time, where the error is the standard deviation. e The linear reflectance spectrum measured after $1 \mathrm{~h}$ of the repeated irradiation for the area inside (red) and outside (blue) the irradiation beam spot. A black dashed line denotes the spectrum measured before the irradiation

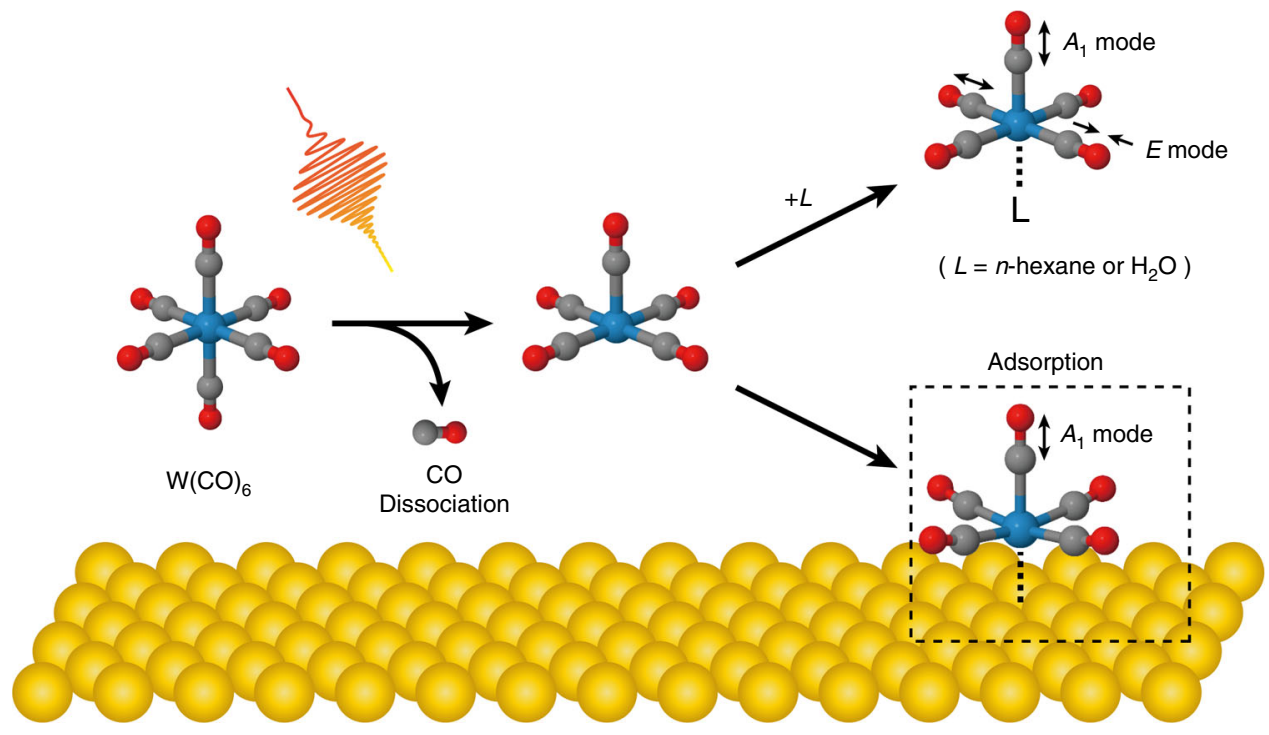

Fig. 5 Proposed reaction schemes induced by antenna-enhanced vibrational ladder climbing. Upon multi-quantum vibrational excitation, the W(CO) 6 molecule loses one CO ligand. The CO dissociation is followed by adsorption on the gold surface or coordination with the solvent molecule. The former species is sensitively detected, while the latter is undetectable in our antenna-enhanced spectroscopy

absorption feature is smeared out because of the broad inhomogeneous linewidth.

CO dissociation induced by chirp-pulsed near-fields. Upon the irradiation of down-chirped pump pulses, we observe VLC up to $v=6$, whose energy is sufficient for the first bond dissociation ${ }^{41,42}$. This suggests that multi-photon excitation energy is channeled into a W-C bond via intramolecular mode-coupling, resulting in dissociation of a CO ligand (see Fig. 5). Here, we note that the energy corresponding to the $v=1 \rightarrow 6$ transition is sufficient for one $\mathrm{CO}$ dissociation, but insufficient for multiple $\mathrm{CO}$ dissociation ${ }^{42}$. Hence, $\mathrm{W}(\mathrm{CO})_{6}$ can lose only one $\mathrm{CO}$ ligand in each single shot process. Furthermore, because dissociated species recombine on a microsecond timescale in condensed phases ${ }^{43}$, shorter than the pump-pulse interval of $2 \mathrm{~ms}$, multiple CO dissociation is much less probable than the single $\mathrm{CO}$ dissociation. 
Attribution of the final product. Spatial localization of this emerging band within the irradiation beam spot suggests that it originates from adsorbates, i.e., $\mathrm{CO}$ or $\mathrm{W}(\mathrm{CO})_{5}$ adsorbed on the gold surfaces. According to previous studies ${ }^{44-50}, \mathrm{CO}$ can be linearly adsorbed on top of a gold atom at the surfaces, and its stretching frequency varies in the range of $2000-2140 \mathrm{~cm}^{-1}$ depending on the surface condition. This frequency range is much higher than $1930 \mathrm{~cm}^{-1}$. Therefore, we can exclude the possibility of adsorbed $\mathrm{CO}$ as the origin of this absorption band. Note that we do not observe any sign of adsorbed $\mathrm{CO}$ on the linear reflectance spectrum at $2000-2140 \mathrm{~cm}^{-1}$ (see Supplementary Fig. 6).

According to the theoretical calculations based on density functional theory (DFT), $\mathrm{W}(\mathrm{CO})_{5}$ gets stabilized when adsorbed on the gold surface (see Supplementary Note 6 for details) and the fundamental transition frequency for $A_{1}$ mode of adsorbed $\mathrm{W}(\mathrm{CO})_{5}$ is red-shifted by $45 \mathrm{~cm}^{-1}$ compared with that of the $T_{1 u}$ mode of W $(\mathrm{CO})_{6}$. This frequency shift agrees well with the value of $53 \mathrm{~cm}^{-1}$ observed in our experiments. Here, we assign the absorption band observed at $1930 \mathrm{~cm}^{-1}$ to the $A_{1}$ CO-stretching mode of $\mathrm{W}(\mathrm{CO})_{5}$ adsorbed on the gold surface (see Fig. 5). This assignment is in line with the electron-dose experiment for $\mathrm{W}(\mathrm{CO})_{6}$ adsorbed on the gold surface ${ }^{51}$, where the authors observed an absorption band originating from the decarbonylated species at $\sim 55 \mathrm{~cm}^{-1}$ lower frequency than that of the parent molecule. Estimation of reaction efficiency is provided in Supplementary Note 5.

It should be noted that the $\mathrm{W}(\mathrm{CO})_{5}$ molecule adsorbed on the gold surface has a CO-stretching mode of $E$-symmetry as well. This $E$ mode, however, is almost IR-inactive because it violates the surface selection rule, originating from the mirror-image dipole formation ${ }^{52}$. Furthermore, antenna-enhanced spectroscopy is much more sensitive to the $A_{1}$ mode than the $E$ mode because the transition dipole moment of the former is parallel to the surface normal as the plasmonic near-fields.

The gradual blue-shift of this absorption band with the pump irradiation (Fig. 4d) is explained by the dipole-dipole coupling among the adsorbate molecules ${ }^{53,54}$. In the present case, as the coverage with adsorbed molecules increases, the dipole-dipole interaction becomes stronger to induce a blue-shift. We can intuitively understand it by considering an array of interacting dipoles, where the restoring force acting on the oscillating dipoles is increased by the Coulomb force generated by the charge distributions of the neighboring dipoles.

It is also possible that the species dissociated with the pump pulse are coordinated by the solvent molecules. According to a previous study ${ }^{43}, \mathrm{~W}(\mathrm{CO})_{6}$ in $n$-heptane solution loses one $\mathrm{CO}$ ligand upon UV excitation and forms $\mathrm{W}(\mathrm{CO})_{5} \mathrm{~L}$ in several picoseconds. Subsequently, exchange of the solvent molecule with a water molecule occurs in a few hundred microseconds (water is a little impurity in the solvent). The same scenario can happen for the process after detachment of a CO ligand in our case of vibrational excitation, i.e. $\mathrm{W}(\mathrm{CO})_{5}$ forms $\mathrm{W}(\mathrm{CO})_{5}(n$-hexane $)$ and then $\mathrm{W}(\mathrm{CO})_{5}\left(\mathrm{H}_{2} \mathrm{O}\right)$. The free $\mathrm{CO}$ and the $\mathrm{W}(\mathrm{CO})_{5}\left(\mathrm{H}_{2} \mathrm{O}\right)$ molecule, however, is undetectable in our antenna-enhanced spectroscopy because they quickly diffuse into bulk solution (see Supplementary Note 4$)$.

\section{Discussions}

We have demonstrated successful driving of ground-state dissociation of condensed-phase molecules by employing plasmonic near-fields of chirped mid-IR pulses. Both down-chirping and plasmonic field enhancement play essential roles in raising vibrational populations to higher-lying states and in realizing bond dissociation. The gold nanoantennas play an additional role in stabilizing the dissociated species prior to recombination.
These key roles solve the long-standing issues of poor excitation efficiency and diffusional recombination that have hampered vibration-mediated dissociation of condensed-phase molecules. This successful demonstration proves that the combination of ultrafast optics and nano-plasmonics in the mid-IR range is useful for mode-selective, efficient vibrational excitation. The demonstrated scheme can develop into more powerful and versatile techniques by incorporating arbitrary pulse-shaping, welldesigned plasmonic nanostructures, and functions of metal surfaces, paving the way toward controlled ground-state chemistry.

\section{Methods}

Sample fabrication. Gold nanoantenna 2D arrays of rectangular lattice are fabricated on $\mathrm{CaF}_{2}$ substrate $(20 \mathrm{~mm}$ in diameter, $1 \mathrm{~mm}$ in thickness). Electron beam resist (FEP-171) is spin-coated with a thickness of $300 \mathrm{~nm}$, exposed by electron beam, and then developed. Subsequently, a 5-nm-thick Cr-adhesion layer followed by a 100 -nm-thick Au layer are thermally evaporated and lifted-off by acetone.

Spectroscopic measurements. Linear reflection FTIR measurements are performed for linearly polarized light with $2 \mathrm{~cm}^{-1}$ resolution on a Bruker VERTEX 70 $\mathrm{v}$ spectrometer coupled to an IR microscope (HYPERION 3000, Bruker). Knifeedge apertures limit the measuring area to $200 \mu \mathrm{m} \times 200 \mu \mathrm{m}$. Thermal light is incident from the substrate side at a low angle.

Single-color pump-probe spectroscopy is performed using the following setup. A Ti:sapphire regenerative amplifier generates $1 \mathrm{~mJ},<100 \mathrm{fs}$ pulses at a $1-\mathrm{kHz}$ repetition rate. The subsequent optical parametric amplifier $\left(2 \mathrm{~mm}\right.$ thick $\beta-\mathrm{BaB}_{2} \mathrm{O}_{4}$, type II) and the difference frequency generator (2-mm-thick $\mathrm{AgGaS}_{2}$, type II) produce MIR pulses of $4 \mu \mathrm{J}$ energy with a center frequency of $1930 \mathrm{~cm}^{-1}$ and a fwhm bandwidth of $280 \mathrm{~cm}^{-1}$. A small fraction $(\sim 4 \%)$ of the MIR pulses is split off with a wedged $\mathrm{BaF}_{2}$ window to obtain probe and reference pulses, and the remainder is used for the pump. The pump energy is adjusted with a half-wave plate and a wire-grid polarizer. Down-chirped pump pulses are generated by propagation through a $40-\mathrm{mm}$-thick $\mathrm{CaF}_{2}$ crystal. Pump and probe pulses are focused and overlapped spatially onto the sample by means of an off-axis parabolic mirror with an effective focal length of 4 inch, which are linearly polarized in parallel to the longer axes of the nanoantennas. The incidence angle of the pump is $0^{\circ}$, and that of the probe is $<10^{\circ}$. The pump beam with a diameter of $200 \mu \mathrm{m}$ at the sample spatially covers the probe beam spot of $150 \mu \mathrm{m}$ diameter. The pumpinduced change in absorbance (measured in transmission geometry for the antenna-uncoupled case) and that in reflectance (measured in reflection geometry for the antenna-coupled case) are monitored by the probe pulse with a 2-ps time delay, controlled by a mechanical stage. The spectrum of the transmitted/reflected probe pulse is measured by a monochromator and a MCT $2 \times 32$ detector array.

\section{Data availability}

The data that support the findings of this study are available from the corresponding author upon reasonable request.

\section{Code availability}

The Matlab code of the numerical simulations is available from the corresponding author upon reasonable request.

Received: 14 April 2019 Accepted: 9 August 2019 Published online: 29 August 2019

\section{References}

1. Kohler, B. et al. Controlling the future of matter. Acc. Chem. Res. 28, 133-140 (2002).

2. Polanyi, J. C. Some concepts in reaction dynamics. Acc. Chem. Res. 5, 161-168 (1972).

3. Doslić, N. et al. Ultrafast photoinduced dissipative hydrogen switching dynamics in thioacetylacetone. Phys. Chem. Chem. Phys. 1, 1249-1257 (1999).

4. Ohtsuki, Y., Sugawara, M., Kono, H. \& Fujimura, Y. Quantum control of molecular reaction dynamics by laser pulses: development of theory and its application. Bull. Chem. Soc. Jpn 74, 1167-1191 (2001).

5. Artamonov, M., Ho, T.-S. \& Rabitz, H. Quantum optimal control of HCN isomerization. Chem. Phys. 328, 147-155 (2006).

6. Gollub, C. et al. Chemoselective quantum control of carbonyl bonds in Grignard reactions using shaped laser pulses. Phys. Chem. Chem. Phys. 12, 15780-15787 (2010)

7. Gordon, R. J. \& Rice, S. A. Active control of the dynamics of atoms and molecules. Annu. Rev. Phys. Chem. 48, 601-641 (1997). 
8. Maas, D. J. et al. Vibrational ladder climbing in NO by (sub) picosecond frequency-chirped infrared laser pulses. Chem. Phys. Lett. 290, 75-80 (1998).

9. Windhorn, L. et al. Molecular dissociation by mid-IR femtosecond pulses. Chem. Phys. Lett. 357, 85-90 (2002).

10. Witte, T. et al. Controlling molecular ground-state dissociation by optimizing vibrational ladder climbing. J. Chem. Phys. 118, 2021-2024 (2003).

11. Windhorn, L. et al. Getting ahead of IVR: a demonstration of mid-infrared induced molecular dissociation on a sub-statistical time scale. J. Chem. Phys. 119, 641-645 (2003).

12. Kleiman, V. D., Arrivo, S. M., Melinger, J. S. \& Heilweil, E. J. Controlling condensed-phase vibrational excitation with tailored infrared pulses. Chem. Phys. 233, 207-216 (1998).

13. Witte, T., Yeston, J. S., Motzkus, M., Heilweil, E. J. \& Kompa, K. L. Femtosecond infrared coherent excitation of liquid phase vibrational population distributions ( $>$ s). Chem. Phys. Lett. 392, 156-161 (2003).

14. Ventalon, C. et al. Coherent vibrational climbing in carboxyhemoglobin. Proc. Natl Acad. Sci. USA 101, 13216-13220 (2004).

15. Strasfeld, D. B., Shim, S.-H. \& Zanni, M. T. Controlling vibrational excitation with shaped mid-IR pulses. Phys. Rev. Lett. 99, 038102 (2007).

16. Kemlin, V. et al. Transient two-dimensional infrared spectroscopy in a vibrational ladder. J. Phys. Chem. Lett. 7, 3377-3382 (2016).

17. Kühn, O. Multidimensional vibrational quantum dynamics of CO-heme compounds: ultrafast IVR mediated $\mathrm{Fe}-\mathrm{CO}$ bond-breaking after $\mathrm{CO}$ excitation? Chem. Phys. Lett. 402, 48-53 (2005).

18. Gollub, C. et al. Chirp-driven vibrational distribution in transition metal carbonyl complexes. Phys. Chem. Chem. Phys. 9, 369-376 (2007).

19. Falvo, C., Debnath, A. \& Meier, C. Vibrational ladder climbing in carboxyhemoglobin: effects of the protein environment. J. Chem. Phys. 138, 145101 (2013).

20. Nuernberger, P. et al. Femtosecond quantum control of molecular dynamics in the condensed phase. Phys. Chem. Chem. Phys. 9, 2470-2497 (2007)

21. Hartstein, A., Kirtley, J. R. \& Tsang, J. C. Enhancement of the infrared absorption from molecular monolayers with thin metal overlayers. Phys. Rev. Lett. 45, 201-204 (1980).

22. Hatta, A., Ohshima, T. \& Suëtaka, W. Observation of the enhanced infrared absorption of p-nitrobenzoate on Ag island films with an ATR technique. Appl. Phys. A Solids Surf. 29, 71-75 (1982).

23. Osawa, M., Ataka, K.-I., Yoshii, K. \& Nishikawa, Y. Surface-enhanced infrared spectroscopy: the origin of the absorption enhancement and band selection rule in the infrared spectra of molecules adsorbed on fine metal particles. Appl. Spectrosc. 47, 1497-1502 (1993).

24. Neubrech, F. et al. Resonant plasmonic and vibrational coupling in a tailored nanoantenna for infrared detection. Phys. Rev. Lett. 101, 157403 (2008).

25. Adato, R. et al. Ultra-sensitive vibrational spectroscopy of protein monolayers with plasmonic nanoantenna arrays. Proc. Natl Acad. Sci. USA 106, 19227-19232 (2009)

26. Ataka, K., Stripp, S. T. \& Heberle, J. Surface-enhanced infrared absorption spectroscopy (SEIRAS) to probe monolayers of membrane proteins. Biochim. Biophys. Acta 2283-2293, 2013 (1828).

27. Neubrech, F., Huck, C., Weber, K., Pucci, A. \& Giessen, H. Surface-enhanced infrared spectroscopy using resonant nanoantennas. Chem. Rev. 117, 5110-5145 (2017).

28. Donaldson, P. M. \& Hamm, P. Gold nanoparticle capping layers: structure, dynamics, and surface enhancement measured using 2D-IR spectroscopy. Angew. Chem. Int. Ed. 52, 634-638 (2013).

29. Selig, O., Siffels, R. \& Rezus, Y. L. A. Ultrasensitive ultrafast vibrational spectroscopy employing the near field of gold nanoantennas. Phys. Rev. Lett. 114, 233004 (2015).

30. Kusa, F., Morichika, I., Takegami, A. \& Ashihara, S. Enhanced ultrafast infrared spectroscopy using coupled nanoantenna arrays. Opt. Express 25, 12896-12907 (2017).

31. Morichika, I., Kusa, F., Takegami, A., Sakurai, A. \& Ashihara, S. Antennaenhanced nonlinear infrared spectroscopy in reflection geometry. J. Phys. Chem. C 121, 11643-11649 (2017)

32. Gandman, A., Mackin, R., Cohn, B., Rubtsov, I. V. \& Chuntonov, L. Twodimensional Fano lineshapes in ultrafast vibrational spectroscopy of thin molecular layers on plasmonic arrays. J. Phys. Chem. Lett. 8, 3341-3346 (2017).

33. Kusa, F., Echternkamp, K. E., Herink, G., Ropers, C. \& Ashihara, S. Optical field emission from resonant gold nanorods driven by femtosecond midinfrared pulses. AIP Adv. 5, 077138-0771386 (2015).

34. Imasaka, K., Kaji, T., Shimura, T. \& Ashihara, S. Antenna-enhanced high harmonic generation in a wide-bandgap semiconductor $\mathrm{ZnO}$. Opt. Express 26, 21364-21374 (2018).

35. Kraack, J. P. \& Hamm, P. Vibrational ladder-climbing in surface-enhanced, ultrafast infrared spectroscopy. Phys. Chem. Chem. Phys. 18, 16088-16093 (2016).
36. Botan, V., Schanz, R. \& Hamm, P. The infrared-driven cis-trans isomerization of HONO. II: vibrational relaxation and slow isomerization channel. J. Chem. Phys. 124, 234511-234511 (2006).

37. Killelea, D. R., Campbell, V. L., Shuman, N. S. \& Utz, A. L. Bond-selective control of a heterogeneously catalyzed reaction. Science 319, 790-793 (2008).

38. Delor, M. et al. Toward control of electron transfer in donor-acceptor molecules by bond-specific infrared excitation. Science 346, 1492-1495 (2014).

39. Chen, J., Zhou, X., Zhang, Y. \& Jiang, B. Vibrational control of selective bond cleavage in dissociative chemisorption of methanol on $\mathrm{Cu}$ (111). Nat. Commun. 9, 4039 (2018).

40. Stensitzki, T. et al. Acceleration of a ground-state reaction by selective femtosecond-infrared-laser-pulse excitation. Nat. Chem. 10, 126-131 (2018).

41. Ziegler, T., Tschinke, V. \& Ursenbach, C. Thermal stability and kinetic lability of the metal carbonyl bond. A theoretical study on $\mathrm{M}(\mathrm{CO})_{6}(\mathrm{M}=$ chromium, molybdenum, tungsten), $\mathrm{M}(\mathrm{CO})_{5}(\mathrm{M}=$ iron, ruthenium, osmium), and $\mathrm{M}$ $(\mathrm{CO})_{4}(\mathrm{M}=$ nickel, palladium, platinum). J. Am. Chem. Soc. 109, 4825-4837 (1987).

42. Ishikawa, Y.-I. \& Kawakami, K. Structure and infrared spectroscopy of Group 6 transition-metal carbonyls in the gas phase: DFT studies on $\mathrm{M}(\mathrm{CO})_{\mathrm{n}}(\mathrm{M}=$ Cr, Mo, and W; $\mathrm{n}=6,5,4$, and 3). J. Phys. Chem. A 111, 9940-9944 (2007).

43. Greetham, G. M. et al. Time-resolved multiple probe spectroscopy. Rev. Sci. Instrum. 83, 103107 (2012).

44. Kottke, M. L., Greenler, R. G. \& Tompkins, H. G. An infrared spectroscopic study of carbon monoxide adsorbed on polycrystalline gold using the reflection-absorption technique. Surf. Sci. 32, 231-243 (1972).

45. Jugnet, Y., Aires, F. J. C. S., Deranlot, C., Piccolo, L. \& Bertolini, J. C. CO chemisorption on $\mathrm{Au}(110)$ investigated under elevated pressures by polarized reflection absorption infrared spectroscopy and scanning tunneling microscopy. Surf. Sci. 521, L639-L644 (2002).

46. Piccolo, L. et al. The adsorption of $\mathrm{CO}$ on $\mathrm{Au}(111)$ at elevated pressures studied by STM, RAIRS and DFT calculations. Surf. Sci. 566, 995-1000 (2004).

47. Pischel, J. \& Pucci, A. Low-temperature adsorption of carbon monoxide on gold surfaces: IR spectroscopy uncovers different adsorption states on pristine and rough $\mathrm{Au}(111)$. J. Phys. Chem. C 119, 18340-18351 (2015).

48. Chang, S. C., Hamelin, A. \& Weaver, M. J. Dependence of the electrooxidation rates of carbon monoxide at gold on the surface crystallographic orientation: a combined kinetic-surface infrared spectroscopic study. J. Phys. Chem. 95, 5560-5567 (1991).

49. Miyake, H., Ye, S. \& Osawa, M. Electroless deposition of gold thin films on silicon for surface-enhanced infrared spectroelectrochemistry. Electrochem. Commun. 4, 973-977 (2002).

50. Dunwell, M. et al. The central role of bicarbonate in the electrochemical reduction of carbon dioxide on gold. J. Am. Chem. Soc. 139, 3774-3783 (2017).

51. Rosenberg, S. G., Barclay, M. \& Fairbrother, D. H. Electron induced reactions of surface adsorbed tungsten hexacarbonyl $\left(\mathrm{W}(\mathrm{CO})_{6}\right)$. Phys. Chem. Chem. Phys. 15, 4002-4015 (2013).

52. Greenler, R. G., Snider, D. R., Witt, D. \& Sorbello, R. S. The metal surface selection rule for infrared spectra of adsorbed molecules adsorbed on small metal particles. Surf. Sci. 118, 415-428 (1982).

53. Persson, B. N. J. \& Ryberg, R. Vibrational interaction between molecules adsorbed on a metal surface: the dipole-dipole interaction. Phys. Rev. B 24, 6954-6970 (1981).

54. Hollins, P. \& Pritchard, J. Infrared studies of chemisorbed layers on single crystals. Progr. Surf. Sci. 19, 275-349 (1985).

\section{Acknowledgements}

The sample was fabricated at VLSI Design and Education Center (VDEC), The University of Tokyo. The DFT computations were performed using Research Center for Computational Science (Okazaki, Japan). We thank K. Hirakawa and K. Yoshida (Institute of Industrial Science, University of Tokyo) for technical supports in thermal evaporation. We appreciate M. Kobayashi and H. Tanaka (Institute of Industrial Science, University of Tokyo) for technical supports in micro-FTIR measurements. S.A. acknowledges support from JSPS KAKENHI Grant Numbers JP16K13694. K.I. acknowledges support from JSPS KAKENHI Grant Numbers JP17H06375. A.S. appreciates the support of JSPS Research Fellowship for Young Scientists (No. 16J04694) K.M. acknowledges support from JSPS KAKENHI Grant Numbers JP19K15582. I.M. acknowledges the support of Grants-in-Aid for JSPS Research Fellow (No. JP18J11212).

\section{Author contributions}

S.A. designed this research. I.M. performed device fabrications, numerical simulations, and optical measurements. K.M. and K.I. performed DFT calculations. I.M. and S.A. wrote the paper. I.M., K.M., A.S., K.I., and S.A. participated in discussions on this work. 


\section{Additional information}

Supplementary Information accompanies this paper at https://doi.org/10.1038/s41467019-11902-6.

Competing interests: The authors declare no competing interests.

Reprints and permission information is available online at http://npg.nature.com/ reprintsandpermissions/

Peer review information: Nature Communications would like to thank Mario Borunda and other, anonymous, reviewers for their contributions to the peer review of this work.

Publisher's note: Springer Nature remains neutral with regard to jurisdictional claims in published maps and institutional affiliations. (c) (i) Open Access This article is licensed under a Creative Commons Attribution 4.0 International License, which permits use, sharing, adaptation, distribution and reproduction in any medium or format, as long as you give appropriate credit to the original author(s) and the source, provide a link to the Creative Commons license, and indicate if changes were made. The images or other third party material in this article are included in the article's Creative Commons license, unless indicated otherwise in a credit line to the material. If material is not included in the article's Creative Commons license and your intended use is not permitted by statutory regulation or exceeds the permitted use, you will need to obtain permission directly from the copyright holder. To view a copy of this license, visit http://creativecommons.org/ licenses/by/4.0/.

(C) The Author(s) 2019 